\title{
Group-Theoretical Interpretation of the Korteweg-de Vries Type Equations
}

\author{
F. A. Berezin and A. M. Perelomov \\ Institute of Theoretical and Experimental Physics, SU-117259 Moscow, USSR
}

\begin{abstract}
The Korteweg-de Vries equation is studied within the grouptheoretical framework. Analogous equations are obtained for which the manydimensional Schrödinger equation (with nonlocal potential) plays the same role as the one-dimensional Schrödinger equation does in the theory of the Korteweg-de Vries equation.
\end{abstract}

\section{Introduction}

Let $\mathscr{G}$ be an arbitrary Lie algebra, $e_{i}$ be a basis in $\mathscr{G}, C_{i j}^{k}$ be the corresponding structure constants, $\tilde{\mathscr{G}}$ be the space of linear functionals on $\mathscr{G}$. Denote as $\tilde{e}^{i}$ the basis in $\tilde{\mathscr{G}}$ which is dual to $e_{i}$ and as $x_{i}$ the coordinates in $\tilde{\mathscr{G}}$ with respect to the basis $\tilde{e}^{i}$.

In the space $\mathscr{F}$ of infinitely-differentiable functions on $\tilde{\mathscr{G}}$ consider the operation

$$
[f, g]_{\text {P. B. }} \equiv\{f, g\}=\sum C_{j k}^{i} x_{i} \partial^{i} f \cdot \partial^{k} g, \quad \partial^{j}=\partial / \partial x_{j} .
$$

It was shown in [1] (see also [2-4]) that the operation (1) turns the space of the infinitely-differentiable functions on $\mathscr{G}$ into the Lie algebra. It is natural to call it the Poisson bracket algebra associated with the Lie algebra $\mathscr{G}$.

Let $x=\sum x_{i} \tilde{e}^{i} \in \tilde{\mathscr{G}}, y=\sum y^{i} e_{i} \in \mathscr{G}$,

$$
\langle x, y\rangle=\sum x_{i} y^{i} \text {. }
$$

To each function $f(x) \in \mathscr{F}$ and to each $x \in \tilde{\mathscr{G}}$ the element $\nabla f(x) \in \mathscr{G}$ is put into correspondence according to the relation

$$
\left.\frac{d}{d t} f(x+t y)\right|_{t=0}=\langle y, \nabla f\rangle=\sum y_{i} \partial^{i} f, \quad y \in \tilde{\mathscr{G}} .
$$

With the use of the mapping $\nabla$ the Poisson bracket (1) may be rewritten in the coordinate-independent form

$$
\{f(x), g(x)\}=\langle x,[\nabla f, \nabla g]\rangle,
$$

where $[\nabla f(x), \nabla g(x)]$ stands for the commutator in $\mathscr{G}$. 
If there exists a scalar product $(x, y)$ in the algebra $\mathscr{G}$ which is nondegenerate and invariant under the adjoint representation then the space $\tilde{\mathscr{G}}$ may be identified with $\mathscr{G}$ via the relation

$$
\langle\tilde{y}, x\rangle=(y, x),
$$

where $x \in \mathscr{G}, \tilde{y} \in \tilde{\mathscr{G}}, y$ is the element of $\mathscr{G}$ and to be identified with $\tilde{y}$.

The invariance of the scalar product yields the identity

$$
([c, b], a)+(b,[c, a])=0 .
$$

Therefore, in this case

$$
\begin{aligned}
\{f, g\}(x) & =\langle\tilde{x},[\nabla f, \nabla g]\rangle=(x,[\nabla f, \nabla g]) \\
& =-(\nabla g,[\nabla f, x]),
\end{aligned}
$$

where $\tilde{x} \in \tilde{\mathscr{G}}$ is identified with $x$ via (5).

Consider the dynamics created in $\mathscr{G}$ by the Hamiltonian function $H$ and use the identity (7) to transform the equations of motion

$$
\frac{d f}{d t} \equiv \dot{f}=\{H, f\}=(x,[\nabla H, \nabla f])=(\nabla f,[x, \nabla H]) .
$$

Consider the $j$-th coordinate of the element $x=\sum x^{j} e_{j}$ with respect to the basis $e_{j}$ as $f$.

It is evident that for any $y \in \mathscr{G}$

$$
\left(\nabla x^{j}, y\right)=y^{j} .
$$

Therefore $\dot{x}^{j}=\frac{d x^{j}}{d t}=[x, \nabla H]^{j}$. By multiplying this equality by $e_{j}$ and summing we find that

$$
\dot{x}=[x, \nabla H] \text {. }
$$

Therefore the equations of dynamics in $\mathscr{G}$ are always reducible to the special Lax form

$$
\dot{x}=[x, M], \quad M=\nabla H .
$$

In view of the evident analogy with the equations describing the rotation of rigid body around a fixed point, the form (8) of the equations of motion will be called the Euler equations below.

The considerations given above were presented in [5] in connection with the models of Gross-Neveu type. It was noted there that the Korteweg-de Vries equation is also treatable within the same scheme, a infinite-dimensional analog of the real symplectic algebra playing the role of $\mathscr{G}$.

In the present paper we are describing this algebra and are showing how the Korteweg-de Vries equation can be obtained and studied with its aid. Simultaneously we obtain equations analogous to it which have the same relation to the many-dimensional Schrödinger equation (with nonlocal potential) as the Korteweg-de Vries equation to the one-dimensional Schrödinger equation. 
It should be emphasized that in the cases under consideration the dynamics is developed not on the whole orbit but on its intersection with some manifold which has somewhat complex structure.

In terms of this submanifold one can also describe the inverse scattering method for $\mathrm{KdV}$ equation. This will be done elsewhere.

\section{Abstract Symplectic Algebra}

Let $\mathscr{L}$ be a real Hilbert space. In case $\mathscr{L}$ is realized as $\mathscr{L}^{2}(M, d x)$, where $M$ is a set with the measure $d x$, we shall give linear operators in $\mathscr{L}$ by kernels:

$$
(K f)(x)=\int K(x, y) f(y) d y,
$$

$K(x, y)$ may be a function or a distribution.

Denote by $\mathscr{H}$ the direct sum of two copies of the space $\mathscr{L}: \mathscr{H}=\mathscr{L} \oplus \mathscr{L}$. where $\left(f_{j}, f_{j}\right)$ is the scalar product in $\mathscr{L}$.

Linear operators in $\mathscr{H}$ can be naturally written as second order matrices whose elements are operators in $\mathscr{L}$ :

$$
\begin{aligned}
A & =\left(\begin{array}{ll}
A_{11} & A_{12} \\
A_{21} & A_{22}
\end{array}\right), \quad A f=g=\left(\begin{array}{l}
g_{1} \\
g_{2}
\end{array}\right), \\
g_{j} & =\sum_{k} \int A_{j k}(x, y) f_{k}(y) d y .
\end{aligned}
$$

Consider the skew-symmetrical bilinear form in the space $\mathscr{H}$

$$
(f, g)_{S}=\int\left(f_{1} g_{2}-f_{2} g_{1}\right) d x=\int\left(f^{\prime} \tau g\right) d x=(f, \tau g), \quad \tau=\left(\begin{array}{rr}
0 & \mathbb{1} \\
-\mathbb{1} & 0
\end{array}\right) .
$$

Denote as $\operatorname{Sp}(M, \mathbb{R})$ the Lie algebra consisting of real operators in $\mathscr{H}$ that have the common domain of definition and leave the form (11) invariant: $A \subset \operatorname{Sp}(M, \mathbb{R})$ if $A \tau+\tau A^{\prime}=0$ or, equivalently

$$
A_{22}=-A_{11}^{+}, \quad A_{12}^{+}=A_{12}, \quad A_{21}^{+}=A_{21} \text {. }
$$

The asterisk stands for Hermitian conjugation. We shall refer to $\operatorname{Sp}(M, \mathbb{R})$ as the abstract real symplectic algebra. In case $\operatorname{dim} \mathscr{L}=n<\infty$ it reduces to finitedimensional symplectic algebra $\operatorname{Sp}(2 n, \mathbb{R})$.

The elements of $\operatorname{Sp}(M, \mathbb{R})$ can be written in the form

$$
L=\left(\begin{array}{cc}
A & B \\
C & -A^{+}
\end{array}\right), \quad B^{+}=B, \quad C^{+}=C .
$$

Besides the algebra $\operatorname{Sp}(M, \mathbb{R})$ we will consider also the algebra $\operatorname{Sp}^{2}(M, \mathbb{R})$, that consists of Hilbert-Schmidt operators of the form (13). [For an operator $A$ of the form (10) in $\mathscr{H}$ to be a Hilbert-Schmidt operator it is necessary and sufficient that $A_{j k}$ be Hilbert-Schmidt operators in $\mathscr{L}$.]

Note that there exists an invariant scalar product in the algebra $\operatorname{Sp}^{2}(M, \mathbb{R})$. It has the form

$\left(L_{1}, L_{2}\right)=\frac{1}{2} \mathrm{Sp}\left(L_{1} L_{2}\right)$.

Note also that when $K$ is integer and nonnegative one has

$$
L^{2 K} \bar{\in} \operatorname{Sp}(M, \mathbb{R}), \quad L^{2 K+1} \in \operatorname{Sp}(M, \mathbb{R}) \quad \text { and } \quad \operatorname{Sp}\left(L^{2 K+1}\right)=0 .
$$




\section{Abstract Korteweg-de Vries Equation}

Let $H(L)=H(A, B, C)$ be a differentiable function in the algebra $\operatorname{Sp}(M, \mathbb{R})$. Let us realize the space $\mathscr{L}$ in one or another way as $\mathscr{L}^{2}(M, d x)$. In this case $H(L)$ turns into a functional of the kernels $A(x, y), B(x, y)$, and $C(x, y)$ of the operators $A, B$, and $C$, respectively. Denote as $\partial H / \partial A$ the operator in $\mathscr{L}$ defined by the kernel $(\partial H / \partial A)(x, y)=\delta H / \delta A(y, x)$. Define analogously the operators $\partial H / \partial B$ and $\partial H / \partial C$. One may easily see that $\nabla H(L) \in \mathrm{Sp}(M, \mathbb{R})$ has the form

$$
\nabla H=\left(\begin{array}{cc}
\frac{1}{2} \frac{\partial H}{\partial A} & \frac{\partial H}{\partial C} \\
\frac{\partial H}{\partial B} & -\frac{1}{2}\left(\frac{\partial H}{\partial A}\right)^{+}
\end{array}\right), \quad\left(\frac{\partial H}{\partial C}\right)^{+}=\frac{\partial H}{\partial C}, \quad\left(\frac{\partial H}{\partial B}\right)^{+}=\frac{\partial H}{\partial B} .
$$

Consider as $H$ the following function

$$
H=\mathrm{Sp}\left(A^{4}+3 A^{2}(B C+C B)\right) \text {. }
$$

Its gradient, according to the general equation (16), has the form

$$
\nabla H=\left(\begin{array}{rr}
\tilde{A} & \tilde{B} \\
\tilde{C} & -\tilde{A}^{+}
\end{array}\right),
$$

where

$$
\begin{aligned}
& \tilde{A}=2 A^{3}+\frac{3}{2}(B C A+A B C)+\frac{3}{2}(C B A+A C B) \\
& \tilde{B}=\frac{3}{2}\left(A^{2} B+B A^{2}\right)+\frac{3}{2}\left(B A^{+2}+A^{+2} B\right) \\
& \tilde{C}=\frac{3}{2}\left(A^{2} C+C A^{2}\right)+\frac{3}{2}\left(C A^{+2}+A^{+2} C\right) .
\end{aligned}
$$

In accord with (8) the equation of motion in $\operatorname{Sp}(M, \mathbb{R})$ determined by the Hamiltonian (17) has the form

$$
\dot{L}=[L, \nabla H] \text {. }
$$

Consider the manifold $\mathscr{K} C \mathrm{Sp}^{2}(M, \mathbb{R})$ consisting of elements of the form

$$
L=\left(\begin{array}{ll}
A & B \\
C & A
\end{array}\right), \quad A^{+}=-A[B, A]=[B, C]=0 .
$$

Denote temporarily the r.-h. side of (20) as $U=\left(\begin{array}{ll}U_{11} & U_{12} \\ U_{21} & U_{22}\end{array}\right)$. The direct calculation shows that from (21) it follows that $U_{11}=U_{12}=U_{22}=0$. This implies that the manifold $\mathscr{K}$ is invariant under the dynamics (20). The operators $A, B$ are the first integrals while $C$ develops in time: $L(t)=\left(\begin{array}{cc}A & B \\ C(t) & A\end{array}\right), A=$ const, $B=$ const. After calculating the element $U_{21}$ we find that Eq. (20) with the condition (21) are equivalent to the following equation for $C$ :

$$
\begin{aligned}
\dot{C} & =\left[A^{3}, C\right]+3 A[C, A] A+3 B\left[C^{2}, A\right] \\
& =[A[A[A, C]]]-3 B\left[A, C^{2}\right] .
\end{aligned}
$$

Equation (22) will be referred to as the abstract Korteweg-de Vries equation. 
Let us pass from the algebra $\operatorname{Sp}^{2}(M, \mathbb{R})$ to the algebra $\operatorname{Sp}(M, \mathbb{R})$. Denote the operator in the r.-h. side of (18) as

$$
M=\left(\begin{array}{cc}
\tilde{A} & \tilde{B} \\
\tilde{C} & -\tilde{A}^{+}
\end{array}\right),
$$

where $\tilde{A}, \tilde{B}$, and $\tilde{C}$ are given by Eqs. (19).

There is no invariant scalar product in $\operatorname{Sp}(M, \mathbb{R})$. Therefore there does not exist the function $H$ on $\operatorname{Sp}(M, \mathbb{R})$ such that $M=\nabla H$. We may nevertheless consider the equations of motion

$$
\dot{L}=[L, M]
$$

the conditions (21) being meaningful as before. Once they are fulfilled the operators $A$ and $B$ serve as first integrals and Eq. (24) proves to be equivalent to (22).

Note that it follows from (21) that the eigensubspace for $B$ is invariant under the operators $A$ and $C$. Therefore Eq. (22) reduces to the case when $B=\lambda \mathbb{1}, \mathbb{1}$ being the unit operator. Finally, it is reduced to the case $B=\mathbb{1}$ by the substitution $C \rightarrow \lambda^{-1} C$.

\section{Auxiliary Spectral Problem and the First Integrals for the Abstract Korteweg-de Vries Equation}

Consider Eq. (24) and assume that such operators $L_{1}, R$, and $S, \dot{L}_{1}=0$ exist that

$$
-\left[M, L_{1}\right]=\left(L-L_{1}\right) R+S\left(L-L_{1}\right) .
$$

Let the vector function $\Psi(x, t)=\left(\begin{array}{l}\Psi_{1}(x, t) \\ \Psi_{2}(x, t)\end{array}\right)$ satisfy the equation

$$
\left(L-L_{1}\right) \Psi=0,
$$

where $L$ is the same operator as in (24). By differentiating (26) with respect to $t$ and using (24)-(26) one finds

$$
\begin{aligned}
0 & =(L M-M L) \Psi+\left(L-L_{1}\right) \frac{d \Psi}{d t} \\
& =\left(L-L_{1}\right)\left((M+R) \Psi+\frac{d \Psi}{d t}\right) .
\end{aligned}
$$

In other words, the function

$$
\Phi=\left(\frac{d}{d t}+M+R\right) \Psi
$$

also satisfies Eq. (26). We shall see below, that in the case of the conventional Korteweg-de Vries equation relations (25) and (27) create the wellknown method of investigation of this equation by means of the inverse problem of the scattering theory [8].

Let us assume now that the operator $S$ in $(25)$ is equal to $(-R)$

$$
\left[L_{1}, M\right]=\left[L-L_{1}, R\right] \text {. }
$$


Consider the theory associated with the algebra $\operatorname{Sp}^{2}(M, \mathbb{R})$. In this case it follows from (28) that the functions

$$
I_{n}=\operatorname{Sp}\left(L-L_{1}\right)^{n}
$$

serve as first integrals for Eq. (24).

Indeed it follows from (28) that another $L-M$ pair should exist and be equivalent to the first one

$$
\tilde{L}=[\tilde{L}, \tilde{M}] \text {, }
$$

where

$$
\tilde{L}=L-L_{1}, \quad \tilde{M}=M+R
$$

whence the above statement follows.

We have considered the spectral problem only in one point. It is more natural to consider this problem with a spectral parameter $\lambda$ included

$$
\tilde{L}(\lambda) \Psi=\left(L-\lambda L_{1}\right) \Psi(\lambda)=0 .
$$

Demand that the Lax equation (30) with $\tilde{L}=L-\lambda L_{1}, \tilde{M}=M+\lambda R$ be fulfilled in this case, too. Then, instead of (28), the two conditions

$$
\left[L_{1}, M\right]=[L, R]
$$

and

$$
\left[L_{1}, R\right]=0
$$

must hold. The number of integrals of motion increases since now

$$
I_{n}(\lambda)=\operatorname{Sp}\left(L-\lambda L_{1}\right)^{n}=\sum I_{n, k} \lambda^{n-k}
$$

and the quantities $I_{n, k}(k=0,1, \ldots, n)$ are integrals of motion. Note that this trick was first used in [6].

We are going to show now that with any operator $L_{1}$, irrespective of relation (28), the functions $I_{n}(\lambda)$ (respectively $I_{n, k}$ ) are in involution. This is a consequence of a general circumstance that was as a matter of fact first pointed in [6] (see also [7]).

Let $\mathscr{G}$ be an arbitrary Lie algebra with invariant scalar product, $P(x), Q(x)$ be functions on $\mathscr{G}$ invariant under the adjoint representation, $\lambda_{1}, \lambda_{2}$ be arbitrary numbers, $a \in \mathscr{G}$. In this case

$$
\left\{P\left(x+\lambda_{1} a\right), Q\left(x+\lambda_{2} a\right)\right\}=0
$$

Proof. Let $f(x)$ be an arbitrary function on $\mathscr{G}, y \in \mathscr{G}$

$$
\begin{aligned}
\left.\frac{d}{d t} f(\exp (t \cdot \operatorname{ad}(y)) \cdot x)\right|_{t=0} & =\sum C_{j K}^{l} y^{j} x^{K} \partial_{l} f \\
& =([y, x], \nabla f)=(y,[x, \nabla f]) .
\end{aligned}
$$

The latter equality follows from (6). In particular, if $f(x)$ is an invariant of the adjoint representation the 1 .h. side is equal to zero for any $y$. Therefore

$$
[x, \nabla f(x)]=0 .
$$


Let $P(x), Q(x)$ be functions invariant under the adjoint representation. From (37), (6) in follows that

$$
\{P(x), Q(x)\}=0 \text {. }
$$

Analogously $\{P(x+a), Q(x+a)\}=0$. Consequently

$$
\left(\mu_{1} x+\mu_{2} y,[\nabla P(x), \nabla Q(y)]\right)=0
$$

for any $\mu_{1}, \mu_{2}$. By replacing $x$ by $x+\lambda, a$ and $y$ by $x+\lambda_{2} a$ in this identity and putting $x=\mu_{1}\left(x+\lambda_{1} a\right)+\mu_{2}\left(x+\lambda_{2} a\right)$ we conclude that

$$
\left(x\left[\nabla P\left(x+\lambda_{1} a\right), \nabla Q\left(x+\lambda_{2} a\right)\right]\right)=0
$$

at $\lambda_{1} \neq \lambda_{2}$. With the aid of the obvious limiting transition one finds that (38) remains valid also if $\lambda_{1}=\lambda_{2}$. Equation (38) coincides with (36) owing to the definition of the Poisson bracket ${ }^{1}$. By setting $P=I_{m}, Q=I_{n}$ in (36) one comes to the necessary result.

Note that from Eq. (37) it follows an important consequence for the $\operatorname{Sp}\left(L^{K}\right)$ type Hamiltonians the equations of motion have the trivial form: $\dot{L}=0$. In case one considers not the $\operatorname{Sp}^{2}(M, \mathbb{R})$-theory but the $\operatorname{Sp}(M, \mathbb{R})$-theory the traces do not exist. Nevertheless, the above consideration does not lose sense completely. It indicates that the first integrals should be looked for in the form (29) [or (35)], with the traces understood, however, in the regularized sense. The specific form of this regularization depends on details. It may be scarcely pointed in general form.

In concluding this section we give a nontrivial solution of the Eqs. (33), (34)

$$
L_{1}=\sigma=\left(\begin{array}{rr}
\mathbb{1} & 0 \\
0 & -\mathbb{1}
\end{array}\right), \quad R=-6\left(\begin{array}{cc}
A^{2} & 0 \\
0 & -A^{2}
\end{array}\right)
$$

[one should use (21) when verifying it].

\section{Classical Korteweg-de Vries Equation as a Particular Case of the Abstract One}

Take the space $\mathscr{L}^{2}\left(\mathbb{R}^{1}, d x\right)$ for $\mathscr{L}$, where $d x$ is the conventional Lebesgue measure on a straight line, and the operators $A=\partial_{x}=\partial / \partial x$ and $B=\mathbb{1}$ for $A$ and $B,(C f)(x)$ $=\int W(x, y) f(y) d y, C^{+}=C^{2}$. After substituting these values of $A, B$, and $C$ into (22)

1 Apart from (36), another essential consequence follows from (37):

$$
[\nabla P(x), \nabla Q(x)]=0 .
$$

This relation is evident for regular $x$, i.e. for those $x$ which obey the following condition: the relations $[a, x]=0,[b, x]=0$ lead to $[a, b]=0$. The theorem is wellknown that if $\mathscr{G}$ is a finite-dimensional Lie algebra with nondegenerate invariant scalar product each its element is a limit of regular ones. Thus the relation $(*)$ via the limiting transition may be extended to all the elements of $\mathscr{G}$.

In the infinite-dimensional case no analogous general theorem is known and thus a verification is needed in every given situation. For example, the theorem retains its power for the algebra $\operatorname{Sp}(M, \mathbb{R})$. The corresponding convergence should be understood in the sense of strong operator topology

2 Note that from this it follows $W(x, y)=W(y, x)$ 
we obtain the equation for $W$ :

$$
\begin{aligned}
\frac{d W(x, y)}{d t}= & \left(\partial_{x}^{3}+\partial_{y}^{3}\right) W(x, y)+3 \partial_{x} \partial_{y}\left(\partial_{x}+\partial_{y}\right) W(x, y) \\
& -3\left(\partial_{x}+\partial_{y}\right) W_{2}(x, y),
\end{aligned}
$$

where $W_{2}(x, y)$ is the iterated kernel:

$$
W_{2}(x, y)=\int W(x, \xi) W(\xi, y) d \xi
$$

[when deriving Eq. (40) from (22)] we used the integration by parts

$$
(C A f)(x)=\int W(x, y) \frac{\partial f}{\partial y} d y=-\int \frac{\partial W(x, y)}{\partial y} f(y) d y .
$$

Equation (40) possesses the property easy to verify by direct calculation: if $W(x, y)=V(x) \delta(x-y)$ the r.-h. side of (40) has he analogous form. In other words, the operators of multiplication by a function form in this ease an invariant manifold. By substituting $W(x, y, t)=V(x, t) \delta(x-y)$ into $(40)$ one finds that $V(x, t)$ obeys the Korteweg-de Vreis equation

$$
\frac{\partial V}{\partial t}=\dot{V}=\partial_{x}^{3} V-6 V \partial_{x} V
$$

The auxiliary spectral problem (26), (32) has the form [we set $\lambda=i k$ in (32)]

$$
\left(\begin{array}{cc}
\partial_{x}-i k & 1 \\
V & \partial_{x}+i k
\end{array}\right)\left(\begin{array}{l}
\Psi_{1} \\
\Psi_{2}
\end{array}\right)=0
$$

Equation (42) is evidently equivalent to the Schrödinger equation with respect to $\Psi_{1}$ :

$$
\left(-\frac{d^{2}}{d x^{2}}+V(x)\right) \Psi_{1}=k^{2} \Psi_{1}
$$

Therefore we fall into the standard channel of the studying of the Korteweg-de Vries equation by the inverse scattering method [8]. .

However one may manage without the reduction to the Schrödinger equation, too.

Let $\Psi=\left(\begin{array}{ll}\Psi_{11} & \Psi_{12} \\ \Psi_{21} & \Psi_{22}\end{array}\right)$ be a matrix solution of Eq. (42). Let $\Psi(x)=U(x) \chi(x)$, where

$$
U(x)=\left(\begin{array}{cc}
1 & 1 \\
+i k-\sqrt{V(x)-k^{2}} & i K+\sqrt{V(x)-k^{2}}
\end{array}\right)
$$

and the square root $\sqrt{V(x)-k^{2}}$ is adopted to have the cut along the positive halfaxis and to satisfy the condition $\sqrt{-1}=+i$. For $\chi(x)$ the equation arises

$$
\frac{d \chi}{d x}=Q \chi, \quad Q(x)=\left(\begin{array}{ll}
q_{11} & q_{12} \\
q_{21} & q_{22}
\end{array}\right)
$$


Let $k^{2}>V(x)$. In this case $q_{22}=\bar{q}_{11}, q_{21}=\bar{q}_{12}$. Consequently, there exists a solution of Eq. (44) with the same property: $\chi=\left(\begin{array}{ll}\chi_{1} & \chi_{2} \\ \bar{\chi}_{2} & \bar{\chi}_{1}\end{array}\right)$. Let us impose the auxiliary asymptotic condition on it $\chi(x) \sim \chi_{0}(x) \sim\left(\begin{array}{cc}e^{+i K x} & 0 \\ 0 & e^{-i K x}\end{array}\right)$ at $x \rightarrow-\infty$. The asymptotic behavior of $\chi(x)$ at $x \rightarrow+\infty$ has the form $\chi(x) \sim \chi_{0}(x) A(k, t), A(, t)$ $=\left(\begin{array}{ll}a & b \\ \bar{b} & \bar{a}\end{array}\right)$. Let us put now $\Psi=U \chi, \Phi=U \tilde{\chi}$ in (27). The matrix $\tilde{\chi}$ satisfies the Eq. (44), like $\chi$. The transparent calculations show that

$$
\tilde{\chi}=\left[\frac{d}{d t}+U^{-1} \frac{d U}{d t}+U^{-1}(M+R) U\right] \chi
$$

whence the asymptotic condition for $\tilde{\chi}$ at $x \rightarrow \infty$ follows:

$$
\tilde{\chi} \sim T \chi_{0}(x)=\chi_{0}(x) T, \quad T=-(i K)^{3}(1+3 \sigma), \quad \sigma=\left(\begin{array}{rr}
\mathbb{1} & 0 \\
0 & -\mathbb{1}
\end{array}\right) .
$$

Therefore, $\tilde{\chi}(x, t)=\chi(x, t) T$ at all $x$. With the use of (45) we conclude that when $x \rightarrow+\infty$

$$
\chi(x, t) \sim\left(\frac{d}{d t}+T\right) \chi_{0} A=\chi_{0} A T .
$$

Hence

$$
\frac{d A}{d t}=-[T, A]=3(i k)^{3}[\sigma, A] .
$$

In other words, $\frac{d a}{d t}=0, \frac{d b}{d t}=6(i k)^{3} \cdot \beta$. Thus, $a(k)$ is an integral of motion ${ }^{3}$.

\section{Many-Dimensional Analogs of the Korteweg-de Vries Equation}

Consider as $\mathscr{L}$ the direct sum of $K$ copies of the space $\mathscr{L}^{2}\left(\mathbb{R}^{n}, d x\right)$, where $d x$ is the usual Lebesgue measure in the $n$-dimensional space $\mathbb{R}^{n}, K>2^{n / 2-1}$. It is natural to represent the elements of $\mathscr{L}$ as $K$-component vector-functions on $\mathbb{R}^{n}$ :

Set

$$
f=\left(\begin{array}{c}
f_{1} \\
f_{2} \\
\ldots \\
f_{K}
\end{array}\right), \quad(f, f)=\sum_{j=1}^{K} \int\left|f_{j}\right|^{2} d^{n} x .
$$

$$
A=\sum_{j=1}^{n} \gamma_{j} \partial_{j}, \quad \partial_{j}=\partial / \partial x_{j}, \quad B=\mathbb{1}
$$

where $\gamma_{j}$ are real spinorial matrices, $\gamma_{j}^{2}=1, \bar{\gamma}_{j}=\gamma_{j}, \gamma_{i} \gamma_{j}+\gamma_{j} \gamma_{i}=0$ at $i \neq j$.

3 Since $L$ is a differential operator equations (29), (35) do not have a direct sense. However $\operatorname{Sp}\left(L-\lambda L_{1}\right)^{n}$ admits the regularization with the aid of the resolvent similar to that considered in [9]. With the r.-h. side of (29), (35) understood in this sense one can obtain the complete set of integrals for the Korteweg-de Vries equation. A separate paper will be devoted to the detailed discussion of these questions 
We define the operator $C$ by the matrix kernel $W(x, y)$ :

$(C f)_{K}(x)=\sum_{j} \int W_{K j}(x, y) f_{j}(y) d y$.

Similarly to the previous case we obtain the equation for $W(x, y)$

$$
\begin{aligned}
\frac{\partial W}{\partial t}= & \left\{\left(\Delta_{x} \vec{\partial}_{x}+\Delta_{y} \overleftarrow{\partial}_{y}\right)+3\left(\Delta_{x} \overleftarrow{\partial}_{y}+\Delta_{y} \vec{\partial}_{x}\right)\right\} W \\
& -3\left(\vec{\partial}_{x}+\overleftarrow{\partial}_{y}\right) W_{2}
\end{aligned}
$$

where

$$
\left(\vec{\partial}_{x} W\right)(x, y)=\sum \gamma_{j}\left(\partial / \partial x_{j}\right) W(x, y), \quad \partial_{y} W(x, y)=\sum_{j}\left(\partial / \partial y_{j}\right) W \cdot \gamma_{j},
$$

and $W_{2}$ has the same meaning as in (40). Unlike the one-dimensional case the operators of multiplication by a function do not constitute an invariant subspace. This is not unexpected since for the many-dimensional Schrödinger equation the differential $L-M$ pair is known not to exist [11]. form

The auxiliary spectral problem (32) with the $L_{1}$-matrix of the form (39) has the

$$
\left(\begin{array}{cc}
\vec{\delta}_{x}-i K & \mathbb{1} \\
W & \vec{\delta}_{x+i K}
\end{array}\right)\left(\begin{array}{l}
\Psi_{1} \\
\Psi_{2}
\end{array}\right)=0,
$$

where $\left(W \Psi_{1}\right)_{K}(x)=\sum \int W_{K j}(x, y) \Psi_{1 j}(y) d y$.

It is evidently equivalent to the Schrödinger equation for $\Psi_{1}$ :

$$
-\Delta \Psi_{1}+W \Psi_{1}=k^{2} \Psi_{1} \text {. }
$$

The expressions (29), (35) for the first integrals of motion admit regularization with the aid of the resolvent with the help of sufficiently large power of the resolvent.

We hope we shall study Eq. (46) in details in future.

\section{Conclusions}

I. The theory of Lie groups is a flexible tool to construct nonlinear equations that possess the Lax pair and, what is more, are Euler equations. At present a long list of equations possessing the Lax pair is known, whereas very few of them are known to have a group origin like the Korteweg de Vries equation does.

If they all appeared possessing this property this would imply the triumph of the natural-phylosophic principle in accordance with which the first integrals of the dynamical systems are always connected with groups, although this connection may be more intimate than it is in the Nöether theorem. In particular, all the arguments given in the present paper remain valid if the initial real space $\mathscr{L}$ is replaced by the complex one. Then, the bilinear form (10) changes into Hermitian one and the requirements of its invariance lead to one of the real versions of the orthogonal algebra. The many-dimensional analogs of the Korteweg-de Vries equations built on its basis have the form (46), as before, but the matrixes $\gamma_{j}$ must not longer be real, although the Hermitian character is retained. In particular, the Pauli matrices are acceptable. 
II. The Korteweg-de Vries equation admits the wellknown Hamilton interpretation in which the role of the phase space is played by the space of functions of one real variable with the exterior form

$$
\omega(f, g)=\int_{-\infty}^{\infty} d x f(x) \int_{-\infty}^{x} g(y) d y .
$$

On the other hand, the Poisson bracket (1) on the orbits of the adjoint representation is nondegenerate and given by the exterior form (see [10])

$$
\omega_{x}(\xi, \eta)=(x,[\xi, \eta]),
$$

where $x$ is a point of the orbit, $\xi, \eta$ are vectors tangent to the orbit in the point $x$.

At the first glance it is natural to expect that the restriction of the form (50) onto the manifold of the Lax operators $L=\left(\begin{array}{cc}\partial_{x} & \mathbb{1} \\ v(x) \delta(x-y) & \partial_{x}\end{array}\right)$ should reproduce the form (49). This is not the case.

III. Within the conventional approach to the Korteweg-de Vries equation all the dynamical systems of the form (24) in the space of Schrödinger operators appear to be Hamiltonian as regards to the exterior form (49). This does not hold for the abstract Schrödinger equation. Here is the simplest example of the operator $M$ that creates dynamics in the space of operators $L$ on the form (21) with $A=\partial, B=1, C=U$ but cannot be represented as $M=\nabla H=\left(\begin{array}{cc}\tilde{A} & \tilde{B} \\ \tilde{C} & \tilde{D}\end{array}\right)$ :

$$
\begin{aligned}
& \tilde{A}=\partial^{5}+\frac{5}{2}\left(U \partial^{3}+\partial U \partial^{2}+\partial^{2} U \partial+\partial^{3} U\right)+5 U \partial U+5\left(\partial^{2} U \partial-\partial U \partial^{2}\right) \\
& \tilde{B}=5 \partial^{4}+10 \partial U \partial \\
& \tilde{C}=\frac{5}{2}\left(U \partial^{4}+\partial^{4} U\right)-5\left(\partial U \partial^{3}+\partial^{3} U \partial\right)+10 \partial^{2} U \partial^{2}+5(U \partial U \partial+\partial U \partial U) \\
& \tilde{D}=\partial^{5}+\frac{5}{2}\left(U \partial^{3}+\partial U \partial^{2}+\partial^{2} U \partial+\partial^{3} U\right)+5 U \partial U-5\left(\partial^{2} U \partial-\partial U \partial^{2}\right) .
\end{aligned}
$$

The corresponding Lax equation $\dot{L}=[L, M]$ is equivalent to the nonlinear evolution equation

$$
\dot{U}=\frac{3}{2} U^{\prime \prime \prime \prime \prime}-5\left(U U^{\prime \prime \prime}+U^{2} U^{\prime}\right)
$$

that is not reducible to higher Korteweg-de Vries equation.

Note added. This paper has already been published as a preprint ITEP No. 156(1978). After this paper has been completed there appeared the papers by M. Adler, Invent. Math. 50, 219-248 (1979) and D. Lebedev and $\mathrm{Yu}$. Manin, preprint ITEP No. 155, 1-15(1978) in which the KdV equation is treated from a similar point of view.

\section{References}

1. Berezin, F.A.: Funct. Anal. Appl. 1, 1 (1967)

2. Vergne, M.: Bull. Soc. Math. de France 100, 301 (1972)

3. Kirillov, A.A.: Uspekhi Mat. Nauk 31, 57 (1976)

4. Bourbaki, N.: Groupes et algebras de Lie. Paris: Hermann 1968 
5. Berezin, F.A.: Commun. Math. Phys. 63, 131 (1978)

6. Manakov, S.V.: Funct. Anal. Appl. 10, 93 (1976)

7. Fomenko, A.S., Misčenko, A.T.: Dokl. Akad. Nauk USSR, 231, 536 (1976); Izv. Acad. Nauk SSSR 42, 408 (1978)

8. Gardner, C., Greene, J., Kruskal, M., Miura, R.: Phys. Rev. Lett. 19, 1095 (1967)

9. Gelfand, I.M., Dikiy, L.A.: Funct. Anal. Appl. 10, 13 (1976); 11, 11 (1976)

10. Kirillov, A.A.: Elementy Teorii Predstavleniy (Elements of the representation theory). Moscow: Nauka 1972

11. Perelomov, A.M.: Lett. Math. Phys. 1, 175 (1976)

Communicated by J. Moser

Received February 20, 1979 